\title{
O PAPEL DO PNEUMOPERITÔNIO NA AVALIAÇÃO DE PARÂMETROS RESPIRATÓRIOS E HEMODINÂMICOS DE RATOS ANESTESIADOS, COM OU SEM INTUBAÇÃO INTRATRAQUEAL
}

\author{
THE ROLE OF PNEUMOPERITONEUM IN THE RESPIRATORY AND HEMODYNAMIC \\ EVALUATION IN ANAESTHETIZED RATS, WITH OR WITHOUT INTUBATION
}

\author{
Flávia Coelho de Souza Botter, TCBC-SP'; Murched Omar Taha, TCBC-SP²; \\ Djalma José Fagundes, TCBC-SP ${ }^{3}$; Anna Tereza Negrini Fagundes ${ }^{4}$
}

\begin{abstract}
RESUMO: Objetivo: Estudar as alterações hemodinâmicas e respiratórias em ratos submetidos ou não ao pneumoperitônio com $\mathrm{CO}_{2}$, sob anestesia com ou sem intubação intratraqueal. Método: Ratos machos ( $\mathrm{n}=40$ ), albinos, com peso médio de $300 \mathrm{~g}$, idade de três meses, foram randonizados em quatro grupos: GA - anestesia com intubação endotraqueal por uma hora e com pneumoperitônio de $4 \mathrm{mmHg}$ de $\mathrm{CO}_{2} \mathrm{~GB}$ - anestesia sem intubação endotraqueal por uma hora e com pneumoperitônio de $4 \mathrm{mmHg}$ de $\mathrm{CO}_{2}$; GC - anestesia sem intubação endotraqueal por uma hora e sem pneumoperitônio; GD - anestesia com intubação endotraqueal por uma hora, sem pneumoperitônio. Foram registrados os valores da pressão arterial média (PAM), pressão parcial de gás carbônico (pCO2), frequiência cardíaca (FC), freqüência respiratória (FR), pressão venosa central (PVC), potencial hidrogeniônico acidose $(\mathrm{pH})$, saturação de oxigênio periférico $(\mathrm{spO} 2)$, bicarbonato $\left(\mathrm{HCO}_{3}^{-}\right)$e saturação de oxigênio no sangue arterial $\left(\mathrm{SO}_{2}\right.$, ). Os dados foram coletados no início do experimento (M0), após 30 minutos (M1) e após 60 minutos (M2). Resultados: Em GA e GC (grupos com pneumoperitônio) ocorreu aumento da PAM, $\mathrm{PCO}_{2}, \mathrm{HCO}_{3}^{-}$, FR, PVC e uma diminuição do $\mathrm{pH}, \mathrm{SO}_{2}, \mathrm{spO}_{2}$ e da $\mathrm{FC}$ em relação aos animais sem pneumoperitônio. Por outro lado a intubação intratraqueal demonstrou atenuar estas alterações nos animais com até uma hora de anestesia. Conclusão: A anestesia geral com intubação endotraqueal associada ao pneumoperitônio de $4 \mathrm{mmHg}$, por um período de uma hora, mostrou vantagens do ponto de vista respiratório e hemodinâmico, em relação aos animais com pneumoperitônio e sem intubação e também sobre aqueles com até quatro horas de anestesia, apesar da intubação (Rev. Col. Bras. Cir. 2005; 32(5): 261-266).
\end{abstract}

Descritores: Pneumoperitônio; Intubação intratraqueal; Ratos; Cirurgia Vídeo-assistida.

\section{INTRODUÇÃO}

O surgimento de uma nova técnica operatória, a cirurgia vídeo-assistida ou vídeocirurgia introduziu um fator complicador a ser considerado nos procedimentos anestésicos: o pneumoperitônio. A insuflação da cavidade peritoneal com dióxido de carbono resulta em alterações funcionais no sistema cárdio-respiratório, especialmente devido ao aumento da pressão abdominal ${ }^{1-4}$. Este produz alterações respiratórias que modificam a pressão da artéria pulmonar, com diminuição do volume pulmonar (indicado pelo decréscimo da tensão de oxigênio arterial). A eventual hipóxia pulmonar e a vasoconstricção podem levar à arritmia cardíaca ${ }^{2}$.

As restrições dos movimentos respiratórios acarretam redução da ventilação alveolar nos lobos pulmonares inferiores e conseqüente hipercapnia. O sistema cardiovascular também é afetado com a diminuição do débito cardíaco, aumento da pressão arterial e aumento da resistência vascular periférica ${ }^{5}$.

Berguer et al. ${ }^{6}$ analisaram os efeitos hemodinâmicos e gasométricos em ratos submetidos ao pneumoperitônio com valores progressivos da pressão de $\mathrm{CO}_{2}$ e constataram à ocorrência de bradicardia e acidose respiratória com as pressões maiores, concluindo que valores pressóricos elevados de pneumoperitônio devem ser evitados.

Dalton et al. ${ }^{7}$ analisaram ratos submetidos à insuflação da cavidade peritoneal com $\mathrm{CO}_{2}$, durante 30 minutos, à pressão de $2 \mathrm{mmHg}$, e concluíram que mesmo sob pneumoperitônio de baixa pressão, ocorrem diversas alterações nos parâmetros cárdio-respiratórios.

Windberger et al. ${ }^{8}$ analisaram as alterações hemodinâmicas e metabólicas em suínos submetidos à anestesia geral com pneumoperitônio e relataram um aumento da PAM e do $\mathrm{PCO}_{2}$, diminuição do pH e manutenção dos valores da saturação de oxigênio.

O pneumoperitônio induzido com $\mathrm{CO}_{2}$, para os procedimentos vídeo laparoscópicos, está associado a alterações do débito cardíaco devido às diminuições do retorno venoso ${ }^{6,7}$, do fluxo esplâncnico ${ }^{9}$, da função renal e da ventilação alveolar com conseqüente isquemia e disfunção orgânica ${ }^{8,10}$.

Estes fatores contribuem para a hipercapnia e conseqüente acidose, que, quando discreta, leva à estimulação

1. Médica Veterinária e Pós-Graduanda (Mestrado) da UNIFESP - EPM

2. Professor Afiliado do Departamento de Cirurgia da UNIFESP - EPM.

3. Professor Adjunto do Departamento de Cirurgia e Coordenador do Programa de Pós-Graduação em Cirurgia e Experimentação da UNIFESP - EPM.

4. Acadêmica de Medicina da Universidade de Santo Amaro - UNISA.

Recebido em: 15/04/2005

Aceito para publicação em: 21/09/2005

Conflito de interesse: nenhum

Fonte de financiamento: nenhuma

Trabalho realizado no Programa de Pós-Graduação em Cirurgia e Experimentação - UNIFESP-EPM - São Paulo - SP. 
do sistema simpático com aumento da pressão arterial média, aumento da frequiência cardíaca e aumento da pressão venosa pulmonar ${ }^{2}$. A magnitude da acidose está relacionada com o tempo de duração da distensão abdominal. O aumento do espaço morto ventilatório, determinado pela expansão da cavidade peritoneal durante o pneumoperitônio, e a limitação dos movimentos diafragmáticos, dificultam a eliminação de $\mathrm{CO}_{2}$ pulmonar, levando à acidose respiratória ${ }^{9-11}$.

Os problemas cárdio-respiratórios decorrentes da insuflação da cavidade peritoneal podem ser diminuídos se for realizada intubação intratraqueal, juntamente com ventilação adequada ${ }^{12}$.

O melhor entendimento dos fenômenos fisiopatológicos envolvidos na dinâmica respiratória e suas conseqüentes alterações metabólicas e hemodinâmicas resultantes da associação da agressão anestésica e do pneumoperitônio parece ser um fato relevante a ser investigado.

Este estudo teve como objetivo avaliar alterações provocadas no sistema cardio-respiratório pela associação da anestesia (com ou sem intubação intratraqueal) e o pneumoperitônio.

\section{MÉTODO}

Quarenta ratos (Rattus norvergicus albinus) EPM-1 Wistar, adultos, machos, peso entre 250 e 300 gramas, idade média de três meses, foram acondicionados em gaiolas, recebendo alimentação própria para a espécie e água ad libitum. Antes da realização do experimento foram mantidos em jejum por 12 horas.

Os animais foram distribuídos aleatoriamente em: Grupo A $(n=10)$ - Anestesia com intubação intratraqueal e pneumoperitônio; Grupo B $(n=10)$ - Anestesia sem intubação intratraqueal e pneumoperitônio; Grupo C $(n=10)$ - Anestesia sem intubação intratraqueal e sem pneumoperitônio e Grupo $D(n=10)-$ Anestesia com intubação intratraqueal e sem pneumoperitônio.

Os procedimentos foram realizados segundo as normas técnicas e diretrizes internacionais de pesquisa em animais e após aprovação pela Comissão de Ética em Pesquisa da UNIFESP - EMP (Protocolo n¹422/98).

A anestesia foi realizada com uma associação de cloridrato de cetamina na dose de $40 \mathrm{mg} . \mathrm{Kg}^{-1}$ e cloridrato de xilidino - dihidro - tiazina na dose $10 \mathrm{mg} \cdot \mathrm{Kg}^{-1}$ por via intraperitoneal. Todos os animais permaneceram anestesiados durante uma hora.

Nos animais de todos os grupos foi realizada uma incisão cervical paramediana, dissecados os planos anatômicos, identificadas, isoladas e cateterizadas a artéria carótida e a veia jugular direita. Os cateteres foram conectados a um sistema de monitorização para leitura computadorizada dos parâmetros escolhidos.

Nos animais dos Grupos A e B após antissepsia da parede abdominal com álcool iodado $2 \%$, realizou-se uma incisão mediana de $3 \mathrm{~mm}$, abaixo da cicatriz umbilical, e puncionouse a cavidade abdominal com agulha de Veress. Após verificação da adequada posição da agulha, a cavidade abdominal foi insuflada com $\mathrm{CO}_{2}$, mantendo-se uma pressão constante de $4 \mathrm{mmHg}$.

Nos animais dos grupos A e D foi realizada intubação intratraqueal ${ }^{10}$, enquanto os animais dos grupos B e C permaneceram com respiração espontânea, em ar ambiente.

Os animais foram monitorizados, o que permitiu registrar a pressão arterial média (PAM), pressão venosa central (PVC), saturação total de oxigênio $\left(\mathrm{SpO}_{2}\right)$, freqüência cardíaca (FC) e freqüência respiratória (FR). Os registros foram realizados logo após a cateterização vascular: início dos procedimentos (momento zero - M0), após 30 minutos (momento um - M1) e após 60 minutos (momento dois - M2). Nos mesmos tempos foram coletadas as amostras de sangue $(0,7 \mathrm{ml})$ para dosagem da gasometria arterial, $\mathrm{pH}$, saturação de oxigênio $\left(\mathrm{SO}_{2}\right)$ pressão parcial de dióxido de carbono $\left(\mathrm{pCO}_{2}\right)$ e bicarbonato $\left(\mathrm{HCO}_{3}^{-}\right)$.

Após o término do experimento, com o animal ainda sob efeito anestésico, realizou-se a injeção intracardíaca de cloreto de potássio $19,1 \%$, promovendo a morte do animal.

Para análise dos resultados foram utilizados testes paramétricos e não-paramétricos, conforme a natureza das variáveis estudadas. Análise de variância para mesmos indivíduos ou análise de variância por postos de Friedman para comparar as variáveis estudadas nos momentos zero, $30 \mathrm{mi}-$ nutos e 60 minutos de experimento, em cada um dos grupos do trabalho ${ }^{13}$. Análise de variância para grupos independentes ou análise de variância por postos de Kruskal-Wallis para comparar os grupos em cada um dos momentos do experimento ${ }^{14}$. Fixou-se em 0,05 ou 5\% (a $\left.\leq 0,05\right)$ o nível de rejeição da hipótese de nulidade.

\section{RESULTADOS}

Os resultados são apresentados nas Tabelas 1 a 9 pela média e desvio-padrão da média dos dez animais de cada grupo (A,B, C, e D) e nos três momentos de coleta de dados (MO, M1, M2). Os valores estatisticamente significantes estão assinalados com asteriscos.

As medidas da pressão arterial média no grupo com pneumoperitônio sem intubação (B) foram maiores que no grupo com pneumoperitônio com intubação (D), com trinta (M1) e sessenta (M4) minutos de observação (Tabela 1).

$\mathrm{O}$ potencial hidrogeniônico $(\mathrm{pH})$ teve uma diminuição discreta de valores nos grupos com pneumoperitônio, sem significância estatística (Tabela 2),

A pressão parcial de dióxido de carbono foi maior no grupo com pneumoperitônio sem intubação (B), aos trinta (M1) e sessenta minutos (M2) de observação (Tabela 3),

Quanto ao bicarbonato o grupo sem intubação (B) teve os maiores valores nos dois períodos do experimento (Tabela 4),

A saturação de oxigênio arterial e periférica foi estatisticamente maior em nos dois períodos estudados no grupo com intubação (D) em relação ao grupo sem (B) (Tabelas 5 e 8),

A freqüência cardíaca foi maior no grupo sem intubação e com pneumoperitônio (B) aos trinta e sessenta minutos de observação (Tabela 6).

A freqüência respiratória foi maior no grupo sem 
intubação (B), comparando-se com os outros grupos (A, C e D), em todos os momentos (Tabela 7),

Os valores da pressão venosa central foram maiores nos grupos com pneumoperitônio quando comparados aos demais grupos (Tabela 9),

\section{DISCUSSÃO}

O aumento da pressão abdominal causada pelo pneumoperitônio sem o respaldo de um suporte ao sistema respiratório leva ao aumento da pressão arterial média ${ }^{11}$. Junghans et al. ${ }^{15}$ mostraram que o gás carbônico, diferente do argônio ou hélio, está associado ao aumento pressão arterial e da pressão venosa central. Dalton et al. ${ }^{7}$ mostraram que o $\mathrm{CO}_{2}$, até trinta minutos de anestesia, causa hipertensão arterial leve e reversível, podendo agravar com tempos mais prolongados porém sempre de modo reversível. Rademaker et al. ${ }^{16}$ demonstraram que a pressão arterial e a resistência arterial, assim como a saturação arterial e periférica de oxigênio estão prejudicadas durante a insuflação de gases quer seja o $\mathrm{CO}_{2}$ ou $\mathrm{NO}_{2}$, porém o $\mathrm{CO}_{2}$ leva à alterações menos intensas e duradouras.

Os resultados da pesquisa são concordantes com a literatura, pois os animais com pneumoperitônio e sem intubação traqueal tiveram um aumento significativo da pressão arterial média (Tabela1). O tempo maior de intubação não mostrou influência sobre a magnitude do aumento da pressão arterial média.

Houve uma tendência à acidose nos tempos consi-

Tabela 1 - Valores das médias e desvios-padrões da PAM (pressão arterial média), em mm de Hg, nos três tempos e quatro grupos de estudo.

\begin{tabular}{cccccccccccc}
\hline \multicolumn{3}{c}{ GrupoA } & \multicolumn{3}{c}{ Grupo B } & \multicolumn{3}{c}{ GrupoC } & \multicolumn{3}{c}{ GrupoD } \\
\hline M0 & M1 & M2 & M0 & M1 & M2 & M0 & M1 & M2 & M0 & M1 & M2 \\
101,3 & 91,7 & 100,8 & 110,6 & $122,1^{*}$ & $124^{* *}$ & 125,4 & 114,7 & 108,9 & 103,3 & 107,3 & 106 \\
19 & 21 & 9,7 & 20,7 & 20,3 & 28,6 & 29 & 17,7 & 18,2 & 22,9 & 12,3 & 12,8 \\
\hline
\end{tabular}

Teste de Friedman $(p<0,05)$

* p calculado $=1,37$

$* *$ p calculado $=1,61$

Tabela 2 - Valores das médias e desvios-padrões do pH (potencial hidrogeniônico), nos três tempos e quatro grupos de estudo.

\begin{tabular}{ccccccccccccc}
\hline \multicolumn{3}{c}{ GrupoA } & \multicolumn{4}{c}{ Grupo B } & \multicolumn{3}{c}{ Grupo C } & \multicolumn{3}{c}{ GrupoD } \\
\hline M0 & M1 & M2 & M0 & M1 & M2 & M0 & M1 & M2 & M0 & M1 & M2 \\
7,2 & 7,1 & 7,1 & 7,1 & 7,1 & 7,1 & 7,2 & 7,2 & 7,1 & 7,3 & 7,2 & 7,2 \\
0,05 & 0,06 & 0,07 & 0,09 & 0,01 & 0,09 & 0,05 & 0,04 & 0,09 & 0,04 & 0,01 & 0,08 \\
\hline
\end{tabular}

Teste de Friedman $(p<0,05)$

$p=$ valores não significantes

Tabela 3 - Valores das médias e desvios-padrões de pCO2 (pressão parcial de gás carbônico), em mmHg, nos três tempos e quatro grupos de estudo.

\begin{tabular}{rccccccccccc}
\hline \multicolumn{3}{c}{ GrupoA } & \multicolumn{3}{c}{ Grupo B } & \multicolumn{3}{c}{ Grupo C } & \multicolumn{3}{c}{ GrupoD } \\
\hline M0 & M1 & M2 & M0 & M1 & M2 & M0 & M1 & M2 & M0 & M1 & M2 \\
47,8 & 50,1 & 50,7 & 75,9 & $88,7^{*}$ & $85,7^{* *}$ & 42,7 & 42 & 48,7 & 46,6 & 49,2 & 54,8 \\
9,6 & 6,5 & 7,5 & 15,7 & 20,3 & 21,7 & 5,5 & 5,2 & 9,6 & 9,6 & 21,4 & 14,3 \\
\hline
\end{tabular}

Teste de Friedman $(p<0,05)$

* p calculado $=3,85$

$* *$ p calculado $=3,97$

Tabela 4 - Valores das médias e desvios-padrões de $\mathrm{HCO}_{3}^{-}$(bicarbonato em mmol/L), nos três tempos e quatro grupos de estudo.

\begin{tabular}{ccccccccccccc}
\hline & GrupoA & & \multicolumn{3}{c}{ GrupoB } & \multicolumn{3}{c}{ GrupoC } & \multicolumn{3}{c}{ GrupoD } \\
\hline M0 & M1 & M2 & M0 & M1 & M2 & M0 & M1 & M2 & M0 & M1 & M2 \\
23 & 22 & 21,5 & 26,2 & $25,9 *$ & $25,6^{* *}$ & 19,2 & 18,1 & 17,6 & 21,4 & 18,8 & 21,7 \\
3,5 & 3,9 & 2,8 & 1,5 & 1,4 & 1,5 & 1,6 & 2 & 2,2 & 2,5 & 4,4 & 1,5 \\
\hline
\end{tabular}

Teste de Friedman $(p<0,05)$

* p calculado $=4,94$

** p calculado $=4,94$ 
Tabela 5 - Valores das médias e desvios-padrões de SO2 (saturação de oxigênio de sangue arterial em \%), nos três tempos e quatro grupos de estudo.

\begin{tabular}{cccccccccccc}
\hline \multicolumn{3}{c}{ GrupoA } & \multicolumn{3}{c}{ Grupo B } & \multicolumn{3}{c}{ GrupoC } & \multicolumn{3}{c}{ GrupoD } \\
\hline M0 & M1 & M2 & M0 & M1 & M2 & M0 & M1 & M2 & M0 & M1 & M2 \\
94,3 & $95,4^{*}$ & $98,6^{* *}$ & 76,9 & 80,3 & 80,4 & 83,8 & 90,3 & 79,2 & 86,3 & 83,4 & 84,3 \\
7,9 & 6,7 & 4,3 & 4,9 & 4,5 & 6,5 & 7,4 & 5,3 & 20,3 & 7,7 & 19,4 & 20,8 \\
\hline
\end{tabular}

Teste de Mann-Wittney $(p<0,05)$

* p calculado $=3,48$

$* *$ p calculado $=3,90$

Tabela 6 - Valores das médias e desvios-padrões de FC (freqüência cardíaca em batimentos por minuto), nos três tempos e quatro grupos de estudo.

\begin{tabular}{cccccccccccc}
\hline & GrupoA & \multicolumn{3}{c}{ Grupo B } & \multicolumn{3}{c}{ GrupoC } & \multicolumn{3}{c}{ GrupoD } \\
\hline M0 & M1 & M2 & M0 & M1 & M2 & M0 & M1 & M2 & M0 & M1 & M2 \\
151,9 & 153,9 & 153,7 & 162,7 & $187^{*}$ & $189^{* *}$ & 199,5 & 197,7 & 195 & 189 & 198,7 & 192,6 \\
38,3 & 42,6 & 40,4 & 37,2 & 39 & 19,4 & 35,5 & 50,3 & 38,9 & 48,6 & 54,8 & 50,6 \\
\hline
\end{tabular}

Teste de Friedman $(p<0,05)$

* p calculado $=4,04$

$* *$ p calculado $=4,06$

Tabela 7 - Valores das médias e desvios-padrões de FR (freqüência respiratória em movimentos por minuto), nos três tempos e quatro grupos de estudo.

\begin{tabular}{cccccccccccc}
\hline & GrupoA & \multicolumn{4}{c}{ Grupo B } & \multicolumn{3}{c}{ GrupoC } & \multicolumn{3}{c}{ GrupoD } \\
\hline M0 & M1 & M2 & M0 & M1 & M2 & M0 & M1 & M2 & M0 & M1 & M2 \\
43,7 & 48,7 & 49,5 & 48,5 & $58^{*}$ & $67^{* *}$ & 33,2 & 35,6 & 36,8 & 34,8 & 38 & 40,6 \\
11 & 7,7 & 13,1 & 8,9 & 9,2 & 9,0 & 1,9 & 4,5 & 6,9 & 8,4 & 4,9 & 5,8 \\
\hline
\end{tabular}

Teste de Friedman $(p<0,05)$

* p calculado $=21,38$

$* *$ calculado $=22,26$

derados, mas os animais conseguiram compensar e mantiveram-se dentro de parâmetros estáveis (Tabela 2), concordando com os relatos da literatura. A gravidade da acidose respiratória em modelo experimental em ratos está associada a aumento progressivo das pressões abdominais, sendo que em pressões maiores que $10 \mathrm{mmHg}$ ela torna-se significante ${ }^{6}$. A acidose é dependente da mecânica respiratória e independente do gás utilizado e pode ser minimizada ou abolida pelo controle da freqüência respiratória e ventilação pulmonar adequada $7,9,12,15$

A pressão parcial de dióxido de carbono foi maior no grupo com pneumoperitônio sem intubação, aos trinta e sessenta minutos de observação (Tabela 3), demonstrando que a falta de perviedade das vias aéreas, juntamente com a distensão da cavidade abdominal por $\mathrm{CO}_{2}$, levou os animais a reterem uma quantidade significativamente maior de $\mathrm{CO}_{2}$ (hipercapnia). A retenção de $\mathrm{CO}_{2}$ é referida com freqüência em procedimentos com o uso de $\mathrm{CO}_{2}$, porém sem maiores conseqüências em condições normais ${ }^{8,16-18}$, no entanto, deve ser considerada em pacientes críticos ${ }^{1}$.

Quanto ao bicarbonato o grupo sem intubação teve os maiores valores em todos os períodos do experimento (Tabela 4), o que demonstrou que houve uma tentativa de com- pensar a acidose relacionada aos valores descritos anteriormente, sugerindo a importância da intubação intratraqueal.

A saturação de oxigênio arterial e periférica foi estatisticamente maior em todos os períodos estudados no grupo com intubação em relação ao grupo sem (Tabelas 5 e 8), demonstrando a importância da intubação na vigência do pneumoperitônio. A saturação de oxigênio, tanto arterial quanto periférica, em modelos experimentais, é relatada por Rademaker et al. ${ }^{16}$, Rishimani et al. ${ }^{11}$ e Tsugawa et al. ${ }^{17}$ como prejudicada quanto maior o tempo de anestesia sob uma pressão padronizada ou quanto maior a pressão num tempo determinado.

A freqüência cardíaca foi maior no grupo sem intubação e com pneumoperitônio aos trinta e sessenta minutos de observação (Tabela 6). Este achado está em acordo com os resultados observados na literatura ${ }^{7,9,12,18}$. Os animais sem pneumoperitônio, com ou sem intubação, apresentaram freqüência cardíaca inicial maior que os com pneumoperitônio e assim se mantiveram nos outros dois períodos mostrando que a insuflação peritoneal leva a uma diminuição da frequiência cardíaca.

A freqüência respiratória foi maior no grupo sem intubação, comparando-se com os outros grupos, em todos os momentos (Tabela 7), confirmando os dados anteriores. $\mathrm{O}$ 
Tabela 8 - Valores das médias e desvios-padrões de $\mathrm{SpO}_{2}$ (saturação de oxigênio periférico em \%), nos três tempos e quatro grupos de estudo.

\begin{tabular}{cccccccccccc}
\hline \multicolumn{3}{r}{ GrupoA } & \multicolumn{3}{c}{ Grupo B } & \multicolumn{3}{c}{ GrupoC } & \multicolumn{3}{c}{ GrupoD } \\
\hline M0 & M1 & M2 & M0 & M1 & M2 & M0 & M1 & M2 & M0 & M1 & M2 \\
81,7 & $86^{*}$ & $87,8^{* *}$ & 76,4 & 70,5 & 76,1 & 77,8 & 78,2 & 80,3 & 80,7 & 80,6 & 84 \\
9,1 & 7,1 & 7,3 & 5,2 & 2,5 & 3,4 & 8,5 & 5,3 & 4,1 & 8,2 & 6,6 & 4,7 \\
\hline
\end{tabular}

Teste de Friedman $(p<0,05)$

* p calculado $=9,47$

** p calculado $=9,46$

Tabela 9 - Valores das médias e desvios-padrões de PVC (pressão venosa central em centímetros de água), nos três tempos e quatro grupos de estudo.

\begin{tabular}{cccccccccccc}
\hline \multicolumn{3}{c}{ GrupoA } & \multicolumn{3}{c}{ Grupo B } & \multicolumn{3}{c}{ GrupoC } & \multicolumn{3}{c}{ GrupoD } \\
\hline M0 & M1 & M2 & M0 & M1 & M2 & M0 & M1 & M2 & M0 & M1 & M2 \\
3,4 & $4,9^{*}$ & $4,9^{*}$ & 4,1 & $6,2^{* *}$ & $5,9^{* *}$ & 3,4 & 3,7 & 3,2 & 3,7 & 3,2 & 3,4 \\
1,4 & 1,7 & 1,2 & 2,0 & 2,6 & 1,6 & 1,6 & 1,2 & 0,9 & 2,0 & 1,3 & 1,8 \\
\hline
\end{tabular}

Teste de Friedman $(p<0,05)$

* p calculado $=14,05$

** $p$ calculado $=14,32$

aumento desta freqüência foi uma tentativa, também, de compensar a acidose respiratória. Os animais que são intubados mantêm uma frequiência respiratória mais estável, como se observou em outros trabalhos experimentais ${ }^{7,12}$.

Os valores da pressão venosa central foram maiores nos grupos com pneumoperitônio quando comparados aos demais grupos (Tabela 9), demonstrando que houve um com- prometimento do retorno venoso devido ao pneumoperitônio, também coincidindo com a literatura ${ }^{1,9,15,18}$.

Com base nos resultados obtidos, pode-se concluir que os animais submetidos à intubação intratraqueal durante a anestesia associada ao pneumoperitônio apresentam os parâmetros cardiovasculares e respiratórios mais estáveis.

\begin{abstract}
Background: To investigate hemodynamic and respiratory changes in rats submitted to $\mathrm{CO}_{2}$ pneumoperitoneum or not, under anesthesia with and without endotracheal intubation. Methods: Male albino rats $(n=40)$, average weight of $300 \mathrm{~g}$, three months aged, were randomized: $G A$ - anesthesia with endotracheal intubation for one hour with $\mathrm{CO}_{2}$ pneumoperitoneum $(4 \mathrm{mmHg}) ; \mathrm{GB}$ - anesthesia without intratracheal intubation for one hour with $\mathrm{CO}_{2}$ pneumoperitoneum (4mmHg); $\mathrm{GC}-$ anesthesia without endotracheal intubation for one hour without pneumoperitoneum; GD - anesthesia with endotracheal intubation for one hour without pneumoperitoneum. Median arterial pressure (MAP- mmHg), heart rate (HR-beat/min), respiratory frequency $(\mathrm{RF}-\mathrm{rm} / \mathrm{min})$, central venous pressure $\left(\mathrm{CVP}-\mathrm{cmH}_{2} \mathrm{O}\right)$, peripheral oxygen saturation $\left(\mathrm{STO}_{2}\right)$, partial $\mathrm{CO}_{2}$ pressure $\left(\mathrm{PaCO}_{2}\right)$, arterial bicarbonate $\left(\mathrm{HCO}_{3}^{-}\right)$, oxygen saturation $\left(\mathrm{SO}_{2}\right)$ and $\mathrm{pH}$ were recorded. Measurements were performed at the begining of the procedure (MO) and after, 30 minutes (M1) and 60 minutes (M2). Results: In GA and GC (groups with $\mathrm{CO}_{2}$ pneumoperitoneum) there was an increase of $\mathrm{MAP}, \mathrm{PaCO}_{2}, \mathrm{HCO}_{3}$, $\mathrm{HR}$, CVP and decrease of $\mathrm{pH}$, $\mathrm{CO}_{2}$, $\mathrm{STO}_{2}$ in comparison with those without pneumoperitoneum. On the other hand, endotracheal intubation attenuated these changings during the first hour of anesthesia. Conclusion: General anesthesia with endotracheal intubation, associated to $\mathrm{CO}_{2}$ pneumoperitoneum $(4 \mathrm{mmHg})$ during one hour, proved advantageous in hemodynamic and respiratory systems, in relation to the animals with pneumoperitoneum without endotraqueal intubation, and to those within four hour of anesthesia, in spite of intubation.
\end{abstract}

Key Words: Pneumoperitoneum; intubation, intratracheal; Rats; Video- Assisted Surgery.

\section{REFERÊNCIAS}

1. Holthausen UH, Nagelschmidt M, Troidl H. CO(2) pneumoperitoneum: what we know and what we need to know. World J Surg.1999;23(8):794-800.
2. Laureano BA, Andrus CH, Kaminski DL. Cardiovascular changes during laparoscopy. In: Rosenthal RJ, Friedman RL, Phillips EH. The pathophysiology of pneumoperitoneum. New York: Springer;1991. p.77-84.

3. Rosenthal RJ, Friedman RL, Phillips EH. Intra-abdominal pressure, intracranial pressure, and hemodynamics: a central 
nervous system-regulated response. In: Rosenthal RJ, Friedman RL, Phillips EH. The pathophysiology of pneumoperitoneum. New York: Springer;1991. p. 85-98.

4. Aguiar AA, Gomes OM. Anestesia experimental. In: Gomes OM. Cirurgia experimental. São Paulo: Manole;1978. p.69-73.

5. Johannsen G, Andersen M, Juhl B. The effect of general anaesthesia on the haemodynamic events during laparoscopy with $\mathrm{CO} 2-$ insufflation. Acta Anaesthesiol Scand.1989;33(2):132-6.

6 .Berguer R, Cornelius T, Dalton M. The optimum pneumoperitoneum pressure for laparoscopic surgery in the rat model. A detailed cardiorespiratory study. Surg Endosc.1997;11(9):915-8.

7 .Dalton M, Hildreth J, Matsuoka T, et al. Determination of cardiorespiratory function and the optimum anesthetic regimen during laparoscopic surgery in the rat model. Surg Endosc.1996;10(3):297-300.

8 . Windberger UB, Auer R, Keplinger F, et al. The role of intraabdominal pressure on splanchnic and pulmonary hemodynamic and metabolic changes during carbon dioxide pneumoperitoneum. Gastrointest Endosc.1999;49(1):84-91.

9 Ho HS, Gunther RA, Wolfe BM. Intraperitoneal carbon dioxide insufflation and cardiopulmonary functions. Laparoscopic cholecystectomy in pigs. Arch Surg.1992;127(8):928-33.

10 .Abrão J, Silva VD, Reis LC, et al. [Development of a homemade laryngoscope for orotracheal intubation in rats]. Acta Cir Bras.1994;9(3):136-41.

11 .Rishimani AS, Gautam SC. Hemodynamic and respiratory changes during laparoscopic cholecystectomy with high and reduced intraabdominal pressure. Surg Laparosc Endosc.1996;6(3):201-4.
12 .Leighton T, Pianim N, Liu SY, et al. Effectors of hypercarbia during experimental pneumoperitoneum. Am Surg.1992;58(12):717-21.

13. Siegel S, Castellan NJ. Nonparametrics statistics. New York: Mc Graw-Hill; 1988.

14. Sokal RR, Rohlf FJ. Biometry. San Francisco: WH Freedman; 1969.

15. Junghans T, Bohm B, Grundel K, et al. Effects of pneumoperitoneum with carbon dioxide, argon, or helium on hemodynamic and respiratory function. Arch Surg.1997;132(3):272-8.

16. Rademaker BM, Odoom JA, de Wit LT, et al. Haemodynamic effects of pneumoperitoneum for laparoscopic surgery: a comparison of $\mathrm{CO} 2$ with $\mathrm{N}_{2} \mathrm{O}$ insufflation. Eur $\mathrm{J}$ Anaesthesiol.1994;11(4):301-6.

17. Tsugawa K, Hashizume M, Migou S, et al. The effect of carbon dioxide pneumoperitoneum on the portal hemodynamics in a portal-hypertensive rat model. Surg Laparosc Endosc Percutan Tech.1999;9(5):338-47.

18. Puri G, Singh $H$. Ventilatory effects of laparoscopic under general anaesthesia. Br J Anaesth.1992;68(2):211-3.

Endereçoparacorrespondência:

Murched Omar Taha

Rua Botucatu , 740

04023-900- São Paulo- SP.

Email: taha@uol.com.br 